\section{Distributed memory}

The distribution and quantity of $\mathrm{CD}^{+}$or $\mathrm{CD} 8^{+}$memory $\mathrm{T}$ cells throughout the body are largely unknown. In Blood, Baltimore and colleagues use a combination of whole-body imaging and flow cytometry to determine the number of memory $\mathrm{T}$ cells in various compartments. After adoptive transfer, $\mathrm{CD}^{+}$or $\mathrm{CD}^{+}$cells initially assume a similar distribution pattern (gut and lung), but during the expansion and contraction phases, when memory cells develop, they coalesce into distinct compartments. Specifically, $\mathrm{CD}^{+} \mathrm{T}$ cells show tropism for peripheral lymph nodes, whereas $\mathrm{CD}^{+} \mathrm{T}$ cells accumulate in the gut. This distribution pattern continues long term ( $>8$ weeks) and is by and large the same whether cells are effector memory or central memory cells or are in any of the helper $T$ cell subsets $\left(T_{H} 1, T_{H} 2\right.$ or $\left.T_{H} 17\right)$. These distributions are a direct consequence of the relative expression of chemokines and integrin receptors on the cells and their trophic cytokine requirements.

\section{ZF}

Blood 118, 3039-3050 (2011)

\section{Inflammasome receptors}

Inflammasomes are large cytoplasmic complexes that sense pathogenassociated and danger signals, but the molecular mechanisms of inflammasome activation are unclear. In Nature, Shao and colleagues show that NAIP proteins function as receptors for bacterial flagellin and type III secretion system (TTSS) proteins to activate the NLRC4 (IPAF) inflammasome. NAIP5 directly and specifically interacts with flagellin, and this promotes NAIP5-NLRC4 association. In an analogous way, NAIP2 serves as a receptor for TTSS rod proteins such as Salmonella PrgJ and Burkholderia BsaK. Human NAIP functions analogously to mouse NAIP2 and NAIP5 in recognizing the TTSS needle subunit (cprI) of Chromobacterium violaceum and triggering NLRC4 inflammasome activation. The inflammasome-activating activities of flagellin and TTSS rod and needle proteins lie in their C-terminal leucine-rich regions, which share structural features.

Nature (15 September 2011) doi:10.1038/nature10510

\section{Autoimmunity and PTPN22}

An R620W polymorphism in the phosphatase PTPN22 is associated with several human autoimmune diseases. In the Journal of Clinical Investigation, Meffre and colleagues determine how this variant can affect the clearance of autoreactive B cells. They find more autoreactive $B$ cells in otherwise healthy homozygous and heterozygous carriers of the PTPN22 variant. Notably, autoreactive antibodies are present in these carriers without signs of any overt autoinflammatory disease. Healthy carriers of this PTPN22 variant also have frequencies of autoreactive $B$ cell clones equivalent to those of patients with type 1 diabetes or rheumatoid arthritis. Microarray analysis of B cells from healthy donors carrying the variant PTPN22 shows generalized upregulation of genes associated with B cell survival, activation and proliferation, including those encoding costimulatory molecules, Toll-like receptors and cytokine receptors. Finally, B cells from carriers of the PTPN22 variant show greater responsiveness to stimulation with the costimulatory molecule CD40.

J. Clin. Invest. 121, 3635-3644 (2011)

\section{Lineage priming}

Transcriptional regulators act in concert to specify distinct developmental stages, but how such networks are established during development remains unclear. In Immunity, Mercer et al. describe the evolving enhancer repertoire during B cell differentiation by long-term culture of hematopoietic stem cells generated by enforcement of expression of the E-protein inhibitor Id2. Induced downregulation of Id2 allows differentiation. The presence of monomethylation of histone $\mathrm{H} 3$ at Lys4 demonstrates that lineage-restricted enhancers are already primed in multipotent progenitors. The enhancers of genes with similar activity are enriched for distinct cis-regulatory codes. For example, genes upregulated during B cell development frequently contain binding sites for the transcription factors PU.1 and Runx at the hematopoietic stem cell stage and for the transcription factors E2A and, ultimately, EBF at the commitment stage, whereas genes repressed in $\mathrm{B}$ cells contained binding sites for transcription factors E2A and Fli in islands of monomethylated histone H3 Lys4 in committed B cells.

Immunity (15 September 2011) doi:10.1016/j.immuni.2011.06.013

\section{Primitive gut interactions}

Protochordates lack adaptive immune receptors and rely instead on the expression of innate recognition receptors (VCBPs) composed of tandem variable (V) domains linked to a chitinbinding domain. In the Proceedings of the National Academy of Science, Dishaw et al. describe the genomic structure and function of VCBP found in Ciona, a sessile urochordate organism. Unlike those in amphioxus, another protochordate, genes encoding VCBPs in Ciona do not show extensive polymorphism in their exons, although there is allelic diversity in intronic regions, and they are not generated by somatic rearrangement. VCBP is expressed in gut and stomach epithelial cells and in vacuoles of granular amebocytes, which have phagocytic activity. Soluble VCBPs are released into the gut lumen, where they recognize and opsonize bacteria via their V domains. Surprisingly, the chitinbinding domain does not contribute to these activities, which raises the issue of the function of this conserved domain in hostmicrobe interactions.

Proc. Natl. Acad. Sci. USA (23 September 2011) doi:10.1073/ pnas. 1109687108

\section{Neural-iNKT communication}

Immunosuppression and ensuing infections, especially aspiration pneumonia, commonly occur after ischemic brain infarcts and contribute substantially to the mortality that can follow a stroke. In Science, Kubes and colleagues identify a neural circuit that connects brain ischemia to alterations in the function of hepatic invariant natural killer T cell ( $i$ NKT cells) that lead to the suppression of adaptive immune responses. In a mouse model of stroke, liver $i \mathrm{NKT}$ cells alter their migration and switch from producing interferon- $\gamma$ and interleukin 12 to producing interleukin 10 . Surprisingly, these changes do not require presentation of glycolipids mediated by the antigen-presenting molecule CD1d but instead are induced by neutrotransmitters, as blocking $\beta$-adrenergic receptors reverses these effects. Likewise, mice treated with neuroinhibitors fail to become immunosuppressed in the stroke model. Administration of the $i$ NKT cell agonist $\alpha$-GalCer likewise reverses the immunosuppression. These findings highlight an intriguing connection between neural circuits activated by brain infarcts and $i$ NKT cells. $L A D$ Science (15 September 2011) doi:10.1126/science.1210301 